\title{
ABCD Autumn Meeting Abstracts
}

\section{The latest $A B C D$ meeting at the RCP in London, November 2015, saw another strong selection of research, service improvement work and clinical cases submitted for presentation. A selection are included here in brief.}

An audit of blood glucose or bm monitoring in acute medical admissions at the Leicester Royal Infirmary

Edwards I et al. Leiceister

A comprehensive admission assessment would be incomplete without a blood glucose measurement. This project aimed to determine if the CBG was measured, documented and given due credence in acute medical admissions at the LRI between October 2014 and January 2015. The study found that overall CBG is poorly documented and there is a risk of missing new cases of diabetes as well as inadequate management of known cases.

The recommendations were as follows;

- Completion of roll-out of Think Glucose to AMU nursing staff

- Development of a portfolio of educational resources for junior doctors

- Re-audit following implementation of resources

- Patients with a CBG >11.1 should have a $\mathrm{HbA}_{1 \mathrm{c}}$ to aide differentiation between stress hyperglycaemia and newly diagnosed diabetes and to inform on previous control if already known to have diabetes.

Management of hypoglycaemia in inpatients in University Hospital Llandough: Impact of implementation of the "Hypo Sticker"

Ershaid D et al. University Hospital Llandough

Hypoglycaemia is a well-recognised complication of the medical management of diabetes mellitus. The aim of this project was to assess the impact of introducing the "hypo-sticker" on the management of hypoglycaemia for medical patients in University Hospital Llandough.

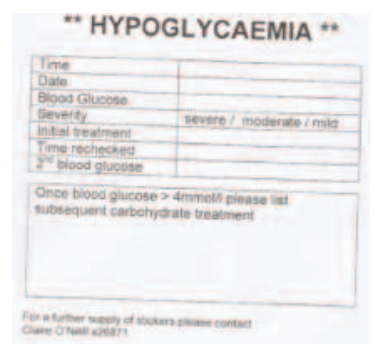

Objectives to be met included the following:

- $100 \%$ hypoglycaemia events are recorded in the medical notes and managed according to treatment guidelines

- $100 \%$ hypoglycaemic events have their blood glucose (BM) checked within 30 minutes of the event being recorded

- $100 \%$ hypoglycaemic events are noted by the medical team at next review.
The authors found that introduction of the "Hypo sticker" has improved documentation as well as both immediate and long term management of hypoglycaemia. More cases are documented in the notes and treated according to guidelines, which has resulted in timely re-measurement of blood glucose (within 30 minutes). This has improved the number of cases that are noted by the medical team on next review, and hence reduction of further hypoglycaemic events.

Evaluating the feasibility of using simulation to teach junior doctors diabetic emergency management Gunganah K et al. Barts Health

Most of the out of the hours management of diabetic emergencies is provided by junior doctors. When junior doctors' understanding and knowledge of diabetes management was assessed by questionnaire, it was found to be consistently poor (Gouveia et al 2011). Historically, this has been addressed by providing small group teaching sessions, case-base discussions and grand round lectures to improve the junior doctors' diabetes management training. However, with the growing popularity for simulation training, we wanted to evaluate whether this teaching method would be effective and preferred by junior doctors.

Simulation training was shown to be a feasible and popular teaching method amongst junior doctors for teaching the management of diabetic emergencies. Future challenges are to increase the junior doctor participation in simulation training and perhaps adapt the session to coincide with trainees' mandatory teaching.

Confidence levels of junior doctors in managing hospital in-patients with diabetes

Livingstone R et al. Glasgow Royal Infirmary.

Lack of confidence in managing diabetes and a need for training has been demonstrated previously in the TOPDOC diabetes study. This local study aimed to evaluate the self-reported confidence levels of junior doctors in managing patients with diabetes and identify training needs. We aimed to get representation from local trainees to clarify whether this remains an issue.

An online pre-validated questionnaire of junior doctors working in the West of Scotland deanery was administered on the original dedicated TOPDOC study web portal, www.topdocdiabetes.org. The researchers found that there is a significant lack of confidence in managing in-patient diabetes. TOPDOC previously demonstrated this across the UK, and we have shown this still exists at a local level. The results should be used to promote efforts to address training needs with diabetologists providing the necessary leadership. 
The effect of the sodium-glucose co-transporter dapagliflozin upon cardiovascular risk factors and risk scores in a Scottish teaching hospital

Mackin S et al.

Sodium-glucose co-transporter (SGLT2) therapies are a fairly new treatment modality and are recognised to have benefits other than improved glycaemic control. They confer the advantage of both weight loss and blood pressure reduction, with potential to improve macrovascular risk as a result. This has recently been demonstrated in the exciting EMPA-REG OUTCOME trial where treatment with Empaglaflozin reduced the primary outcome by $14 \%$.

We sought to ascertain the relative benefits of one of the SGLT2s, dapagliflozin, on cardiovascular risk profile, in a planned, observational analysis of routine care in a university hospital. A total of 94 patients on dapagliflozin were assessed from our clinic population, of which 40 were male. The mean UKPDS 10-year risk for Coronary Heart Disease demonstrated significant reduction after 18-months dapagliflozin treatment, from $20.28 \%$ to $17.39 \%$ ( $p<0.001)$.

We have demonstrated, that similar to previous trial data, dapagliflozin treatment is associated with a reduced $\mathrm{HbA}_{1 c}$, weight loss and reduced blood pressure. As a result, this was associated with a reduction in cardiovascular risk score. This effect requires validation within a randomised control trial with dapagliflozin, and will help determine whether an improvement in cardiovascular outcome is a class effect for this group of medication rather than specific to an individual drug.

Incidence of gestational diabetes mellitus - comparison of old versus new guidelines

Poon-King A et al. University Hospital Llandough

In February 2015, the guidelines regarding the diagnostic criteria for gestational diabetes mellitus were amended, lowering the threshold for diagnosis based on the fasting blood glucose value of oral glucose tolerance test (OGTT). This change was made in part, based on the large double blind study based on the results of the Hyperglycaemia and Adverse Pregnancy Outcome (HAPO) study, which found an association between adverse outcomes even below diagnostic criteria used to identify patients with gestational diabetes mellitus. The aim of this project was to identify how new diagnostic guidelines will have an impact on the incidence of gestational diabetes in the joint diabetes and obstetric department in University Hospital Llandough.

Our study shows that a large majority of oral glucose tolerance tests are done outside the recommended gestation (58\%) with a large majority after 28 weeks gestation.

- Whilst there is an increase in the incidence of gestational diabetes mellitus, actual numbers are quite small. However, the diagnosis of gestational diabetes has major implications of further management for these patients.

- Newly diagnosed gestational diabetics need increased frequency of follow-up (fortnightly) from diabetic specialists and obstetricians, with at least 4 weekly obstetric scans to monitor progress.

- The range of gestational age for the patient's diagnosed varied from 15 weeks gestation to 36 weeks gestation. We estimate that this would have resulted in an additional 50 clinic appointments and 25 obstetric scans over the 13 month period. This will have an impact on departmental resources, availability of clinic slots and clinical case load.

- If all GDM cases had been diagnosed at 26 weeks gestation, this would have resulted in 60 extra clinic appointments and 30 additional obstetric scans

What happened to patients diagnosed with gestational diabetes mellitus in Hastings antenatal clinic? 5 year review Sathiskumar P et al. Conquest Hospital, St. Leonards, East Sussex

We looked at the hospital records of patients with GDM who delivered between 2008 and 2009 and followed their records to see if they developed diabetes over the subsequent years. We used our hospital biochemistry system to search for fasting glucose, OGTT or $\mathrm{HbA}_{1 \mathrm{c}}$ test.

Of 38 patients with GDM, only 18 patients (47\%) had glucose assessments over this 5 year follow up period. Out of this $7(18 \%)$ patients developed diabetes and 1 developed impaired fasting glucose. Only 5 out of this 10 patients had regular monitoring. 20 patients did not have any glucose assessment after their diagnosis of GDM. 5 patients (13\%) had further pregnancy with GDM. We have presented this data to our CCG and planning to adapt yearly $\mathrm{HbA}_{1 \mathrm{c}}$ testing.

Does dapagliflozin affect the metabolic response in patients with elevated alanine aminotransferase (ALT) and Type 2

diabetes?: the Association of British Clinical Diabetologists $(A B C D)$ nationwide dapagliflozin audit Yadagiri $M$ et al. $A B C D$

To evaluate the effect of:

1. dapagliflozin on metabolic response in patients with elevated alanine aminotransferase (ALT).

2. baseline ALT on metabolic response to dapagliflozin.

Collected anonymised data of patients treated with dapagliflozin in the UK

- Patient demographics

- $\mathrm{HbA}_{1 \mathrm{c}}$, weight, ALT

- Diabetes medications

- Adverse events

From 44 centres, 129 contributors provided information on 943 patients. The results showed that apart from a positive impact on glycaemic control and weight, dapagliflozin has a statistically and clinically significant response on ALT reduction in Type 2 diabetes patients with a high baseline ALT $>30 \mathrm{U} / \mathrm{l}$. This result may have may have implications regarding non-alcoholic fatty liver disease, which is associated with Type 2 diabetes. 